\title{
The Effect of Virtual Social Network Based Psycho-Education on Resilience of the Family Caregivers of Clients with Severe Mental Disorders
}

\author{
Naima Seyedfatemi ${ }^{1}$, Masoud Ahmadzad Asl ${ }^{2}$, Raheleh Bahrami ${ }^{3,}$, iD, Hamid \\ Haghani ${ }^{4}$
}

\begin{abstract}
${ }^{1}$ Professor, Nursing Care Research Center, Iran University of Medical Sciences, Tehran, Iran
${ }^{2}$ Assistant Professor, Department of Psychiatry, Tehran Institute of Psychiatry, Faculty of Behavioral Sciences and Mental Health, Iran University of Medical Sciences, Tehran, Iran

${ }^{3}$ MSN, Department of Psychiatric Nursing, Iran University of Medical Sciences, Tehran, Iran

${ }^{4}$ Instructor, Department of Biostatistics, Iran University of Medical Sciences, Tehran, Iran

* Corresponding author: Raheleh Bahrami, MSN, Department of Psychiatric Nursing, Iran University of Medical Sciences, Tehran, Iran. E-mail: r.bahrami12011@gmail.com
\end{abstract}

Received: 21 Feb 2018

Accepted: 06 Nov 2018

\begin{abstract}
Introduction: Psycho-education may positively impact the family caregivers of clients with severe mental disorders and promote in them such positive psychological states as resilience. The purpose of the present study is to examine the effect of virtual social network based psycho-education on resilience of the family caregivers of clients with severe mental disorders.

Methods: The present research is a semi-experimental study in the form of a pre-test and two post-test with two control and test groups conducted in Iran Psychiatric Center in Tehran in 2017. 72 family caregivers of clients with severe mental disorders, with the age of 18 to 65 , participated in the present study. To avoid the spread of the information, the control group was first studied and the test group was studied later. Sampling was conducted in a continuous and non-probable form. Data collection was conducted through demographic questionnaire and Conner-Davidson Resilience Scale (CD-RISC-2003) before the study, immediately after the training, and 4 weeks after that. The virtual training (Psycho-education) was conducted through a telegram channel for four weeks. The data were analyzed by means of SPSS-24 software, and the independent $\mathrm{T}$ tests, couple $\mathrm{T}$, and variances were analyzed by repetitive values.

Results: The results of the study of the demographic features of samples showed that both groups were homogeneous. Based on the independent $\mathrm{T}$ test, the results showed that the change average of resilience score in both test and control groups had meaningful difference statistically, and that the average increased in the test group $(\mathrm{P}<0.001)$. Also, the results of the couple $\mathrm{T}$ test showed that the changes in resilience score in the test group had a meaningful difference statistically, and that the changes in post-test stage were greater than the pre-test stage; they were greater in the second post-test compared to the first post-test and pre-test stages $(\mathrm{P}<0.001)$.

Conclusions: The results of this research showed that the virtual social network based psycho-education promotes the resilience of family caregivers of clients with severe mental disorders. Given the low cost of, and fast access to, social networks, the contents of this training plan can be widely utilized for family caregivers.

Keywords: Psycho-education, Caregiver, Family, Resilience, Virtual Network
\end{abstract}

(c) 2018 Iranian Nursing Scientific Association (INA) 


\section{تأثير آموزش روانشناختى مبتنى بر شبكه اجتماعى مجازى بر تاب آورى مراقبين خانوادتى مددجويان با اختلالهاى روانى شديد}

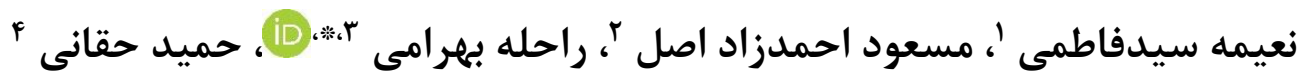

' استاد، مركز تحقيقات يرستارى، دانشخاه علوميزشكى ايران، تهران، ايران

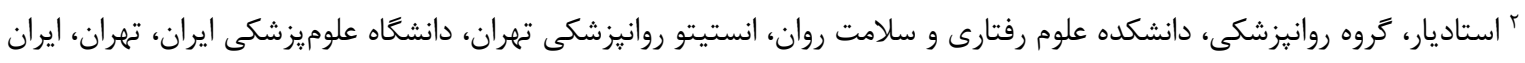

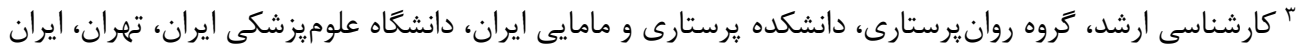

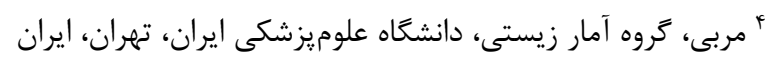

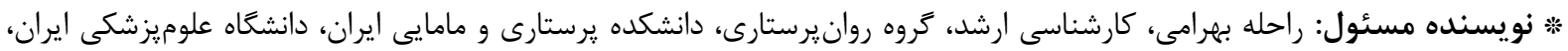
تهران، ايران. ايميل: r.bahrami12011@gmail.com

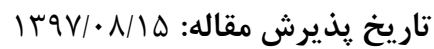

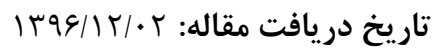

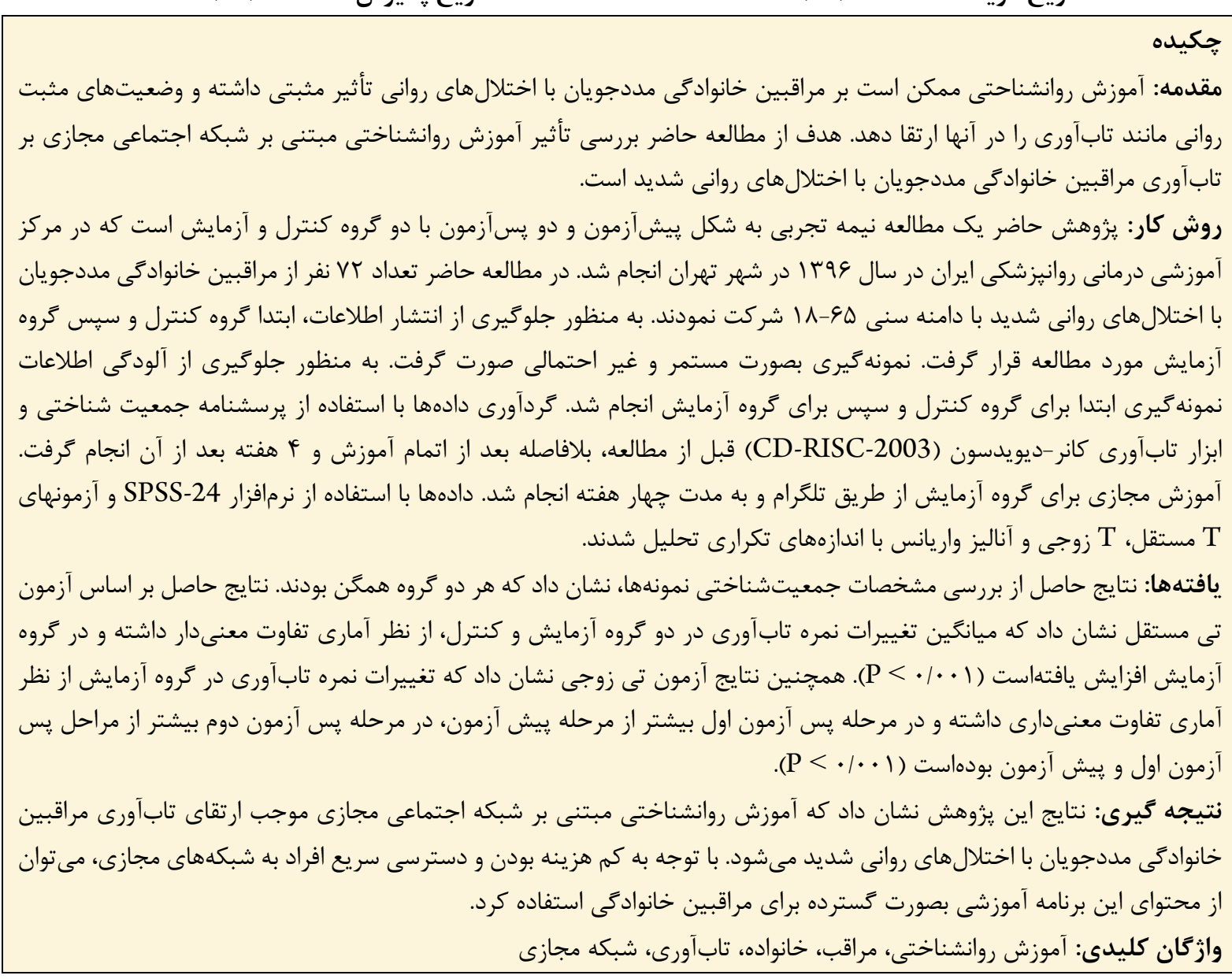

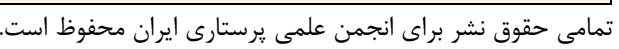

بدعملكردى يا اختلال در فرآيندهاى زيستى، روانى يا رشدى مى باشد إندي

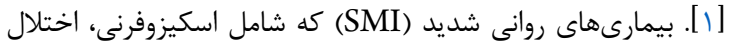

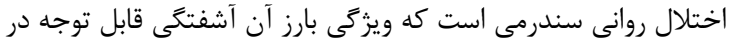

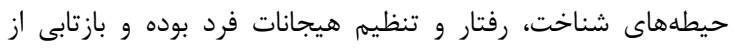


كه مراقبين خانوادگى در روند درمان بيماران دارند، توجه به سلامت و

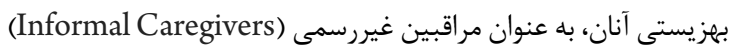

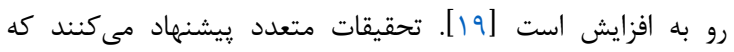

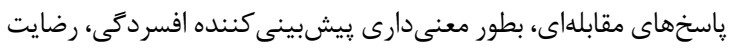

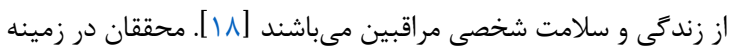

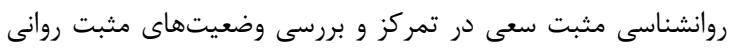

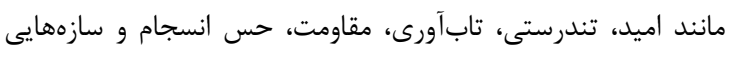

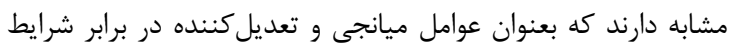

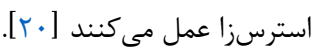

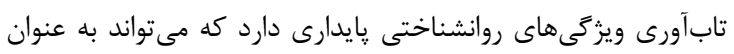

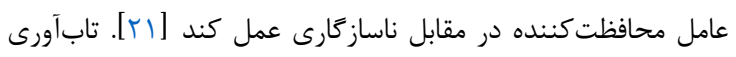

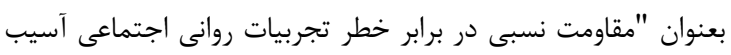

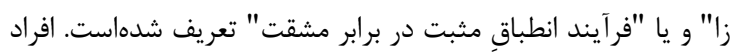

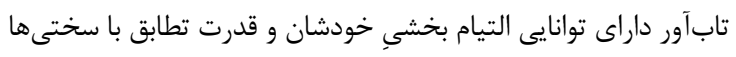

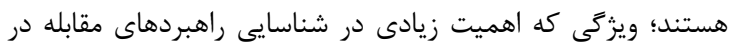

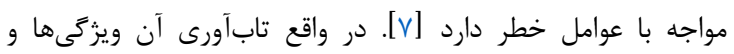

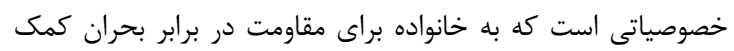

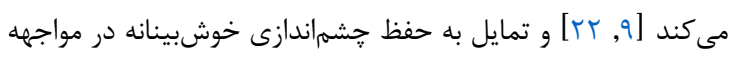

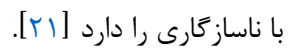

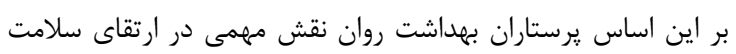

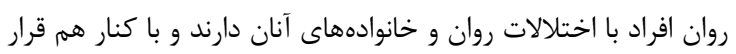

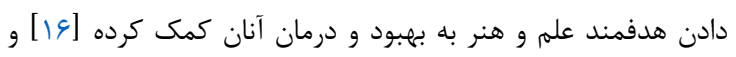

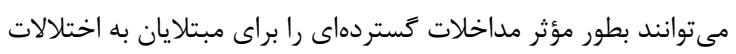

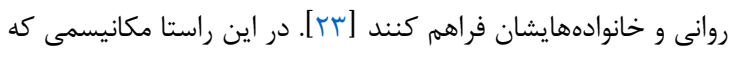

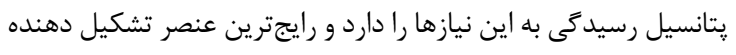

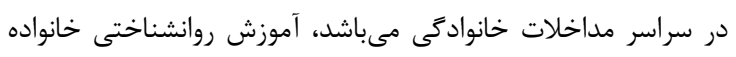

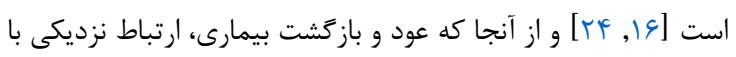

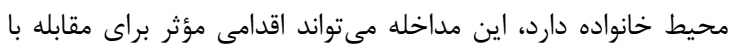

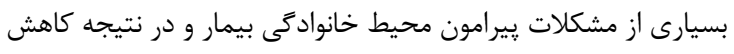

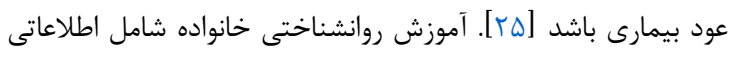

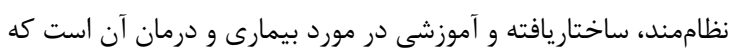

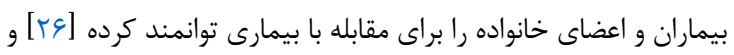

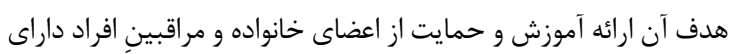

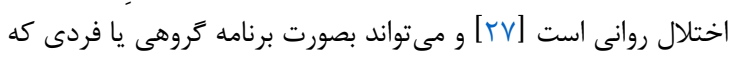

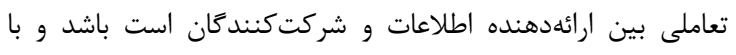

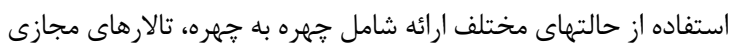

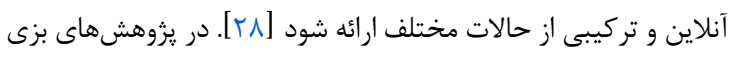

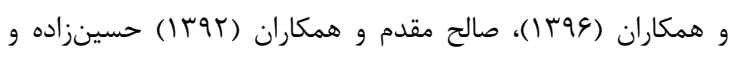

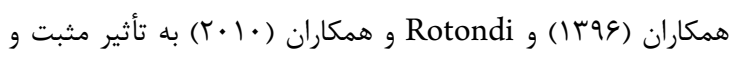

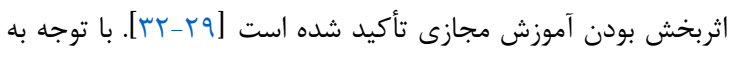

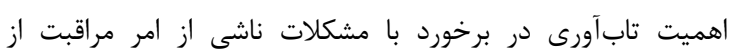

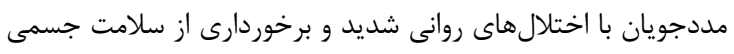

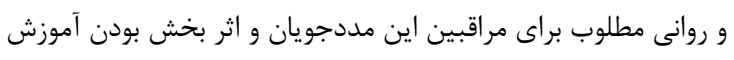

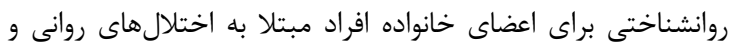

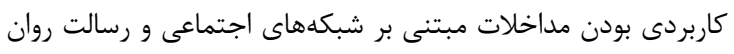

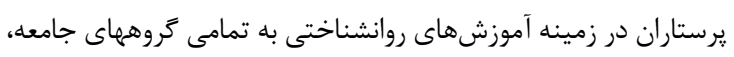

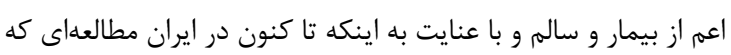

دو قطبى، اختلال افسردىى اساسى و اختلال اسكيزوافكتيو مىباشند،

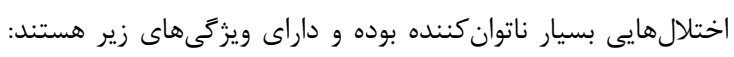

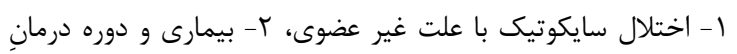

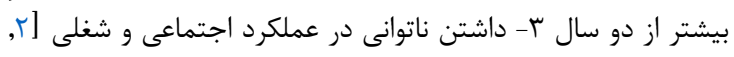

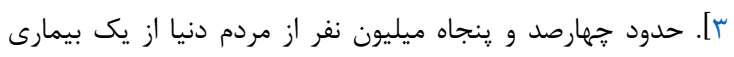

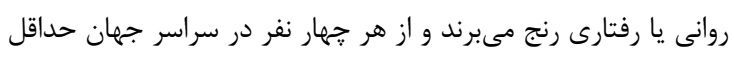

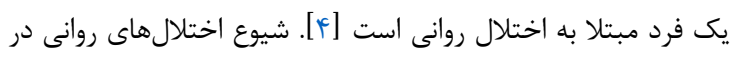

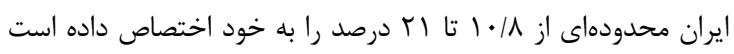

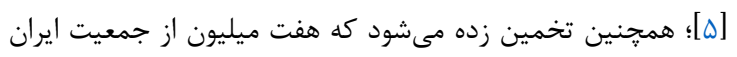

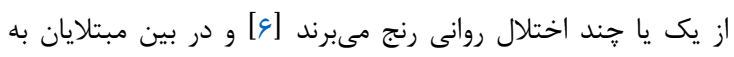

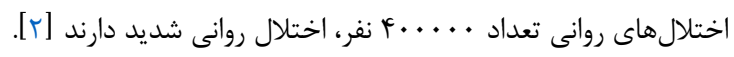

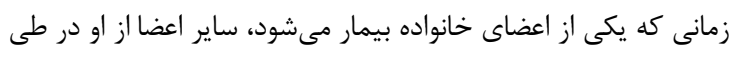

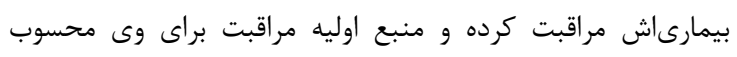

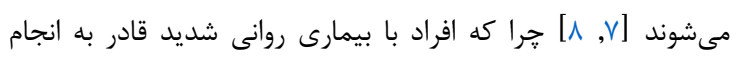

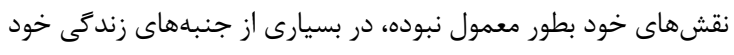

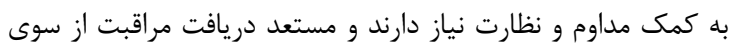

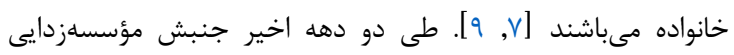
(Deinstitutionalization Policy)

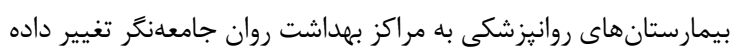

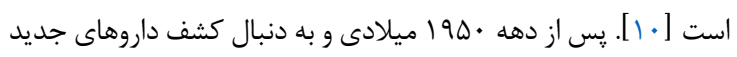

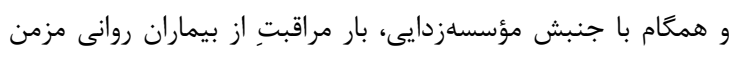

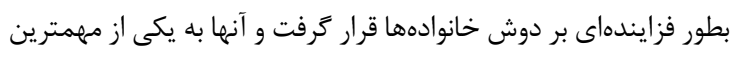

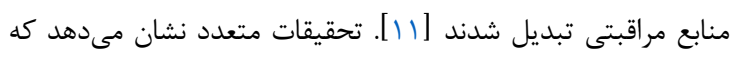

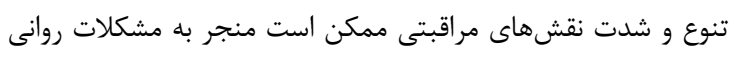

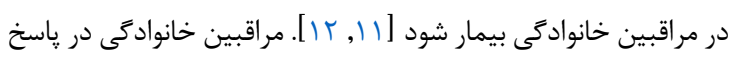

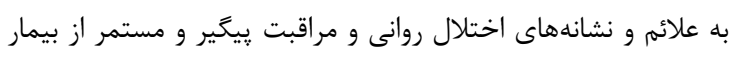

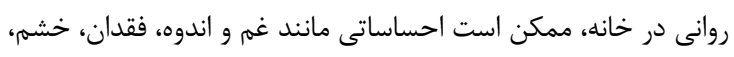

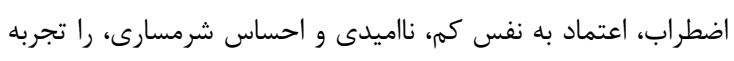

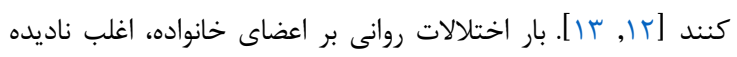

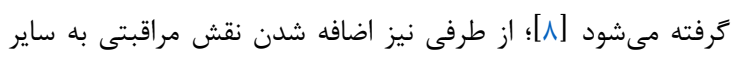

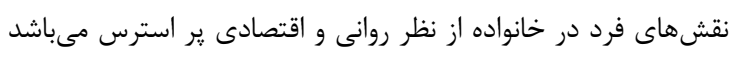

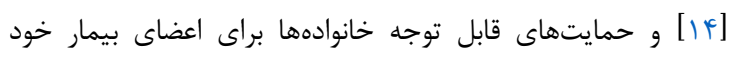

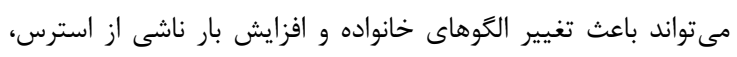

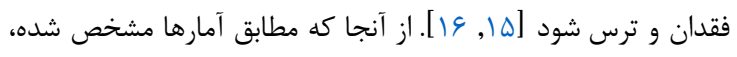

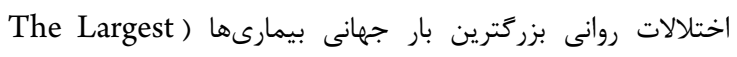
(Global Burden of Disease [ [ ا]؛ درنتيجه نقش مراقبين خانوادىى (Family Caregivers) حائز

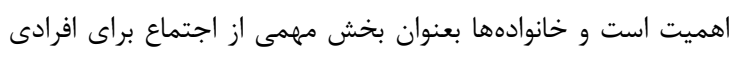

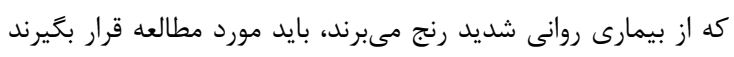

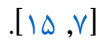

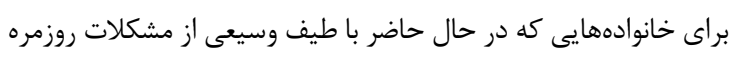

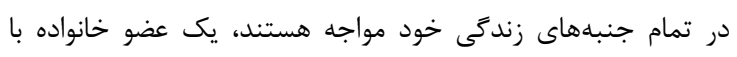

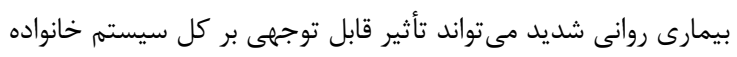

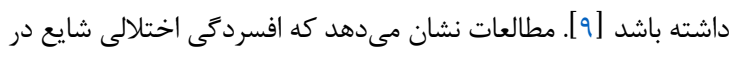

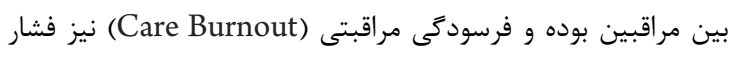

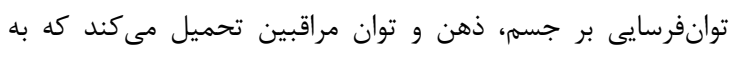

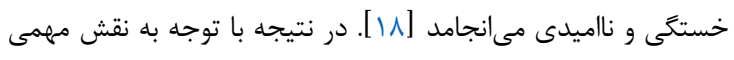


كانالى كه به اين منظور براى خانوادها راهاندازى شده بود، به مدت

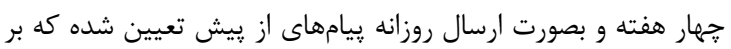

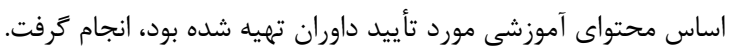

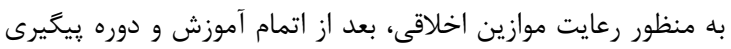

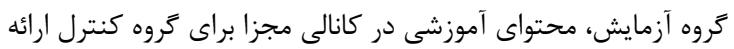

جهت جمع اورى دادهها از يرسشنامه جمعيت شناختى كه شامل

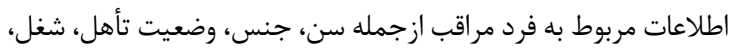

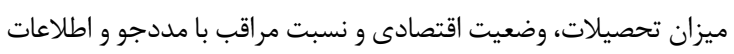

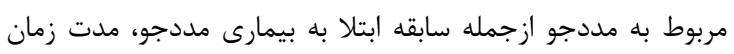

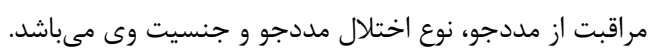

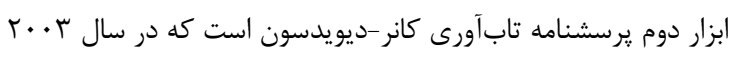

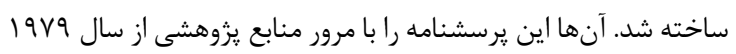

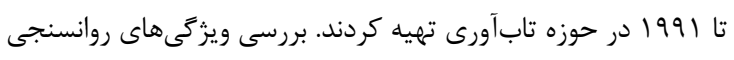

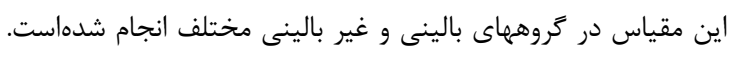

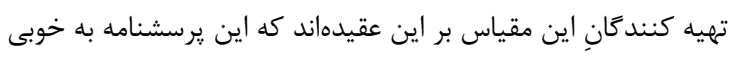

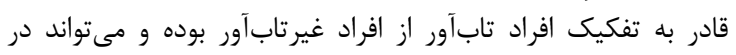

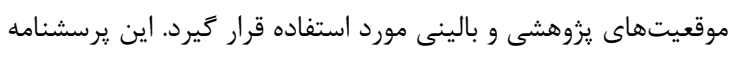

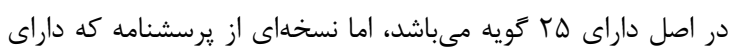

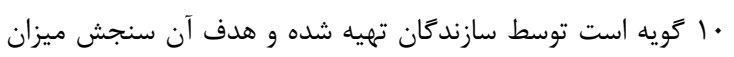

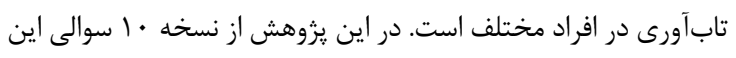

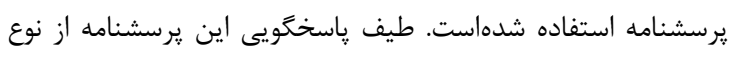

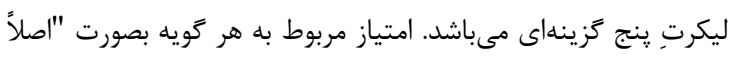

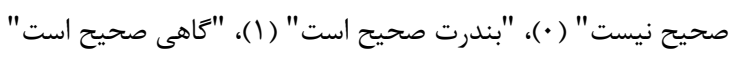

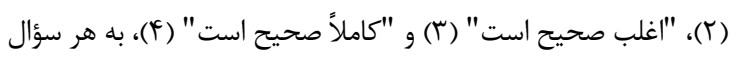

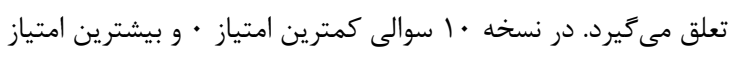

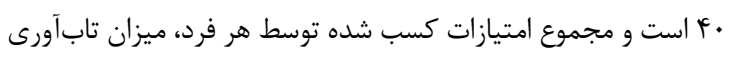

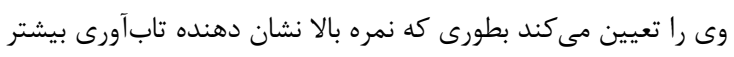

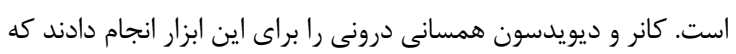

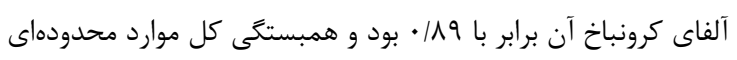

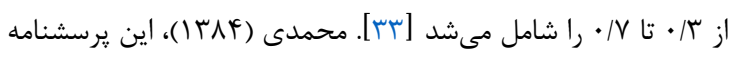

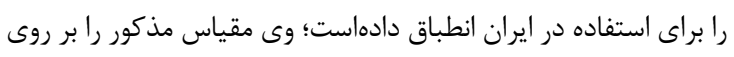

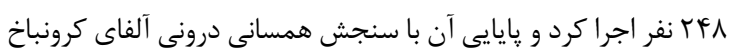

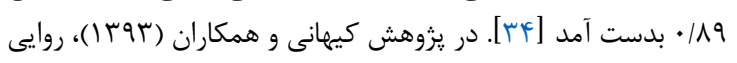

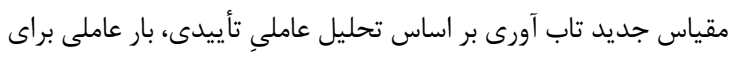

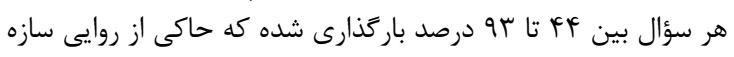

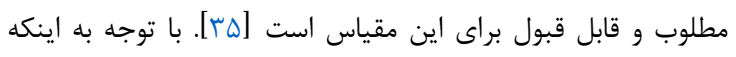

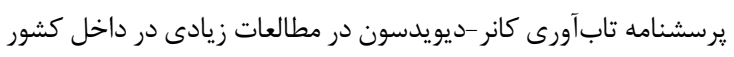

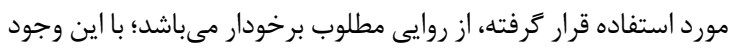

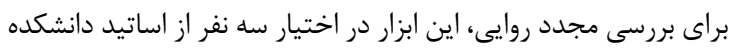

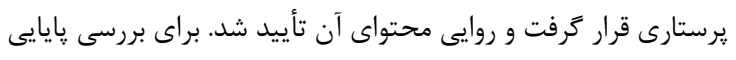

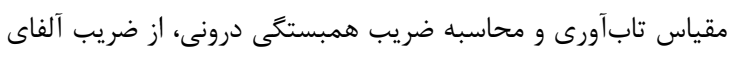

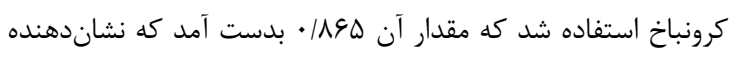
قابل قبول بودن بإيايى درونى اين ابزار است.

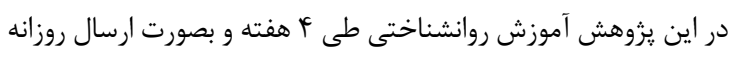

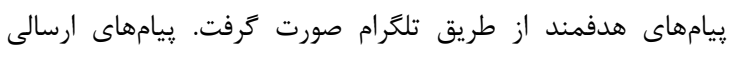

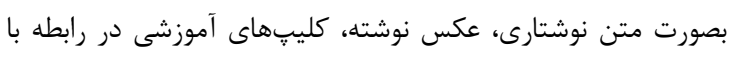

اثربخشى آموزش روانشناختى بر تابآورى مراقبين خانوادكى

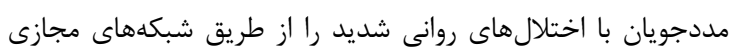

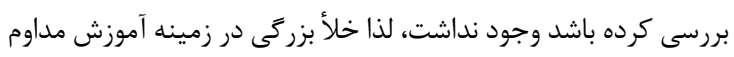

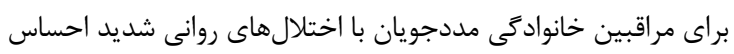

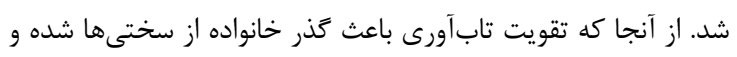

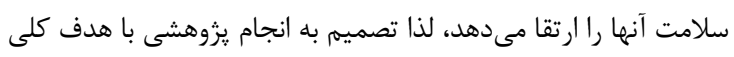

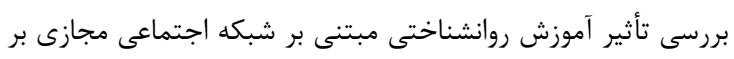

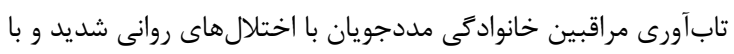

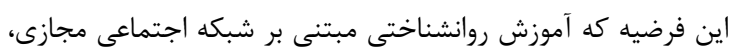

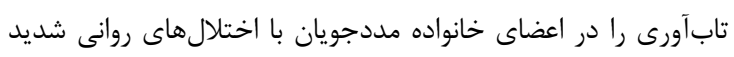
افزايش مىدهد، ترفته شد.

\section{روش كار}

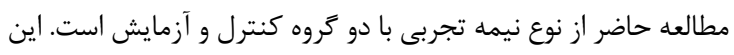

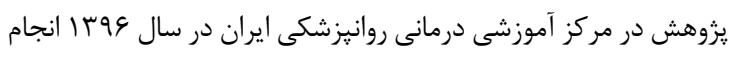

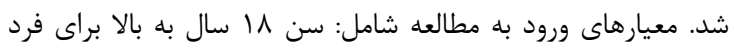

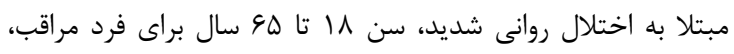

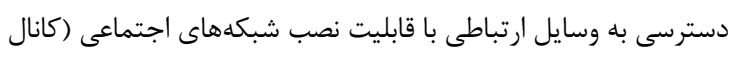

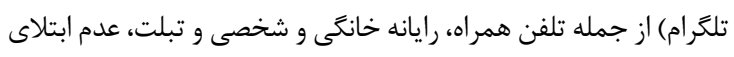

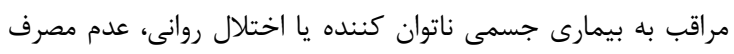

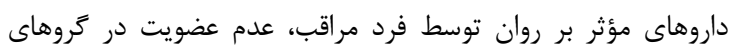

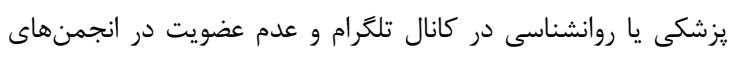

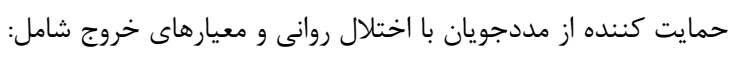

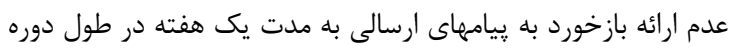

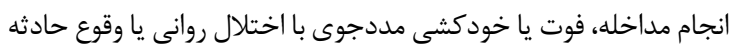

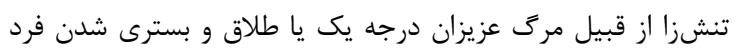

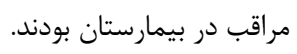

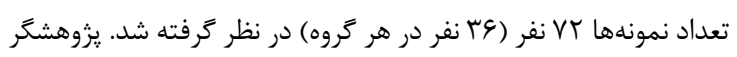

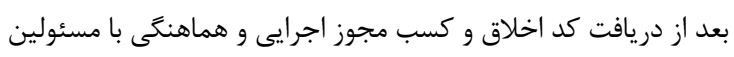

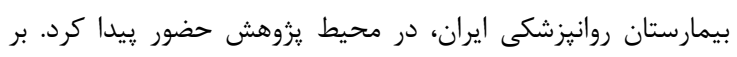

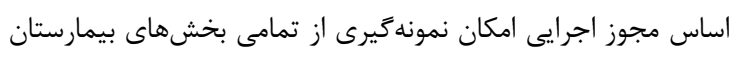

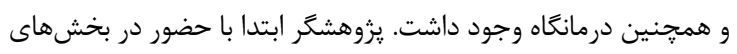

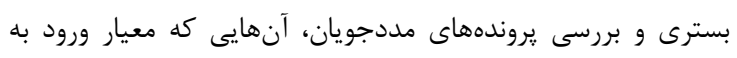

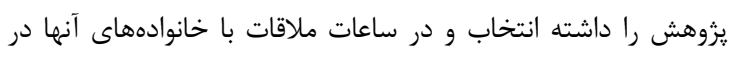

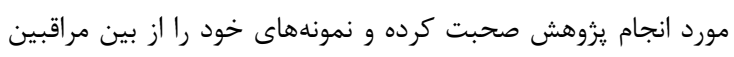

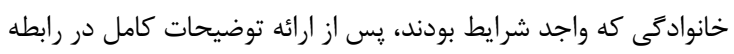

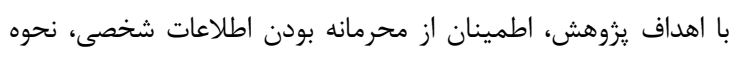

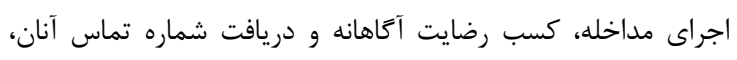

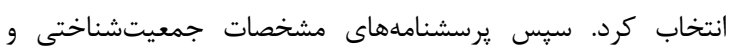

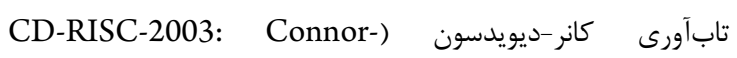
(Davidson resilience scale

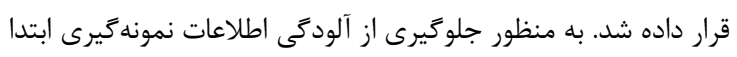

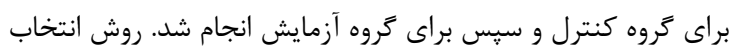

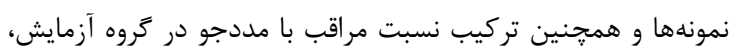

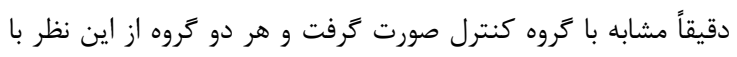

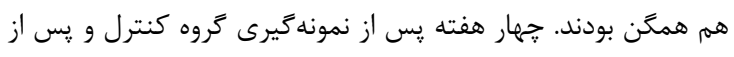

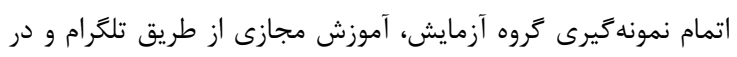


فهرست مطالب آموزشى آن هفته براى آنها ارسال مىشد. عناوينى كه

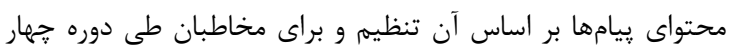
هفتهاى آموزش ارسال شد در جدول آ آمده است.

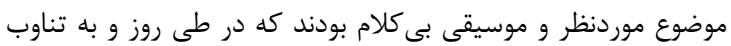

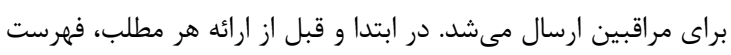

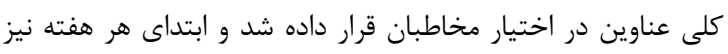

جدول ا: برنامه آموزشى تلكرام در مدت ؟ هفتد

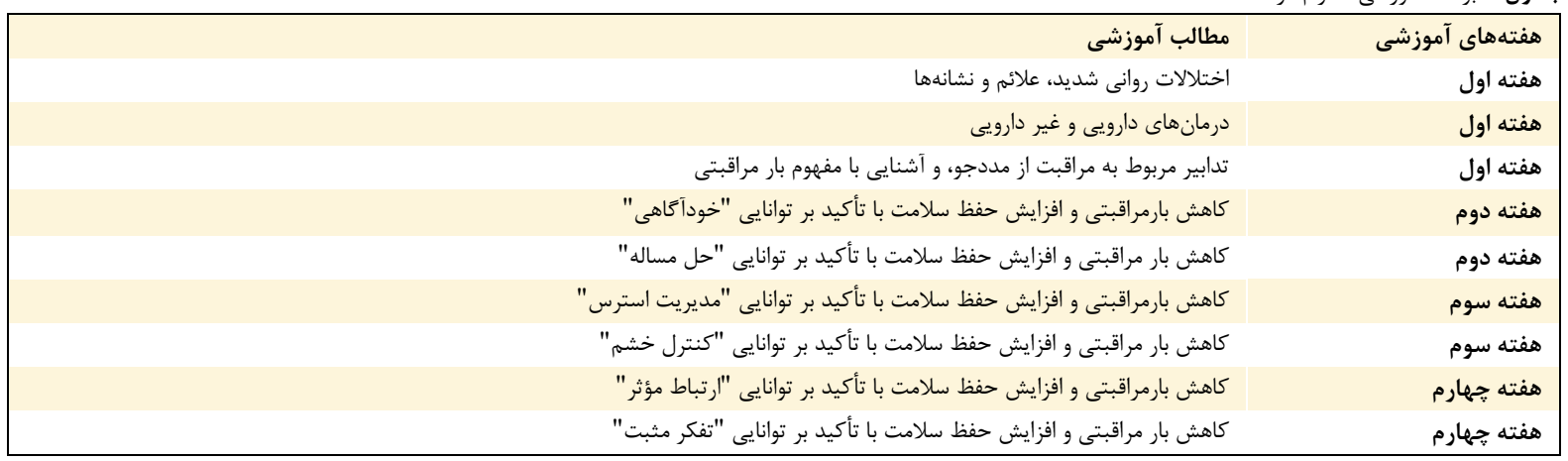

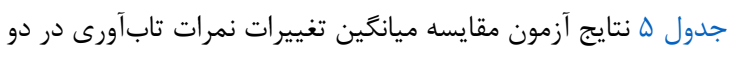

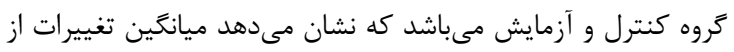

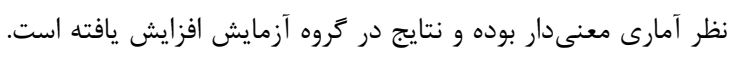

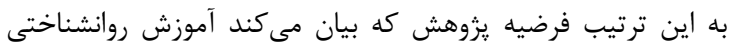

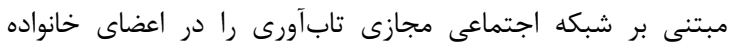

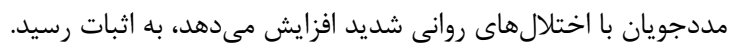

اين مطالعه با هدف كلى "بررسى تأثير آموزش روانشناختى مبتنى بر

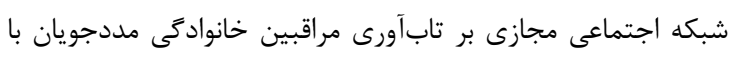

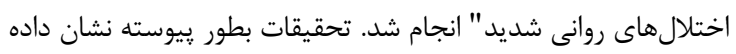

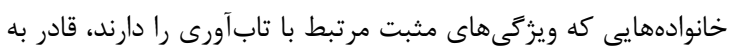

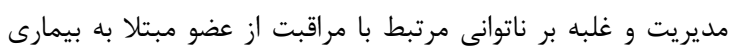

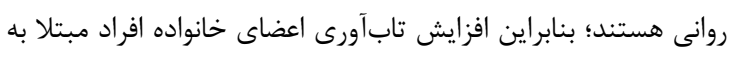

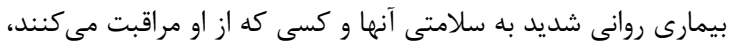

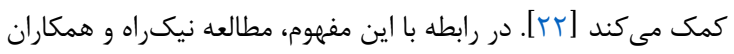

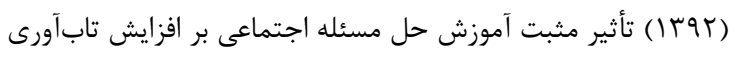

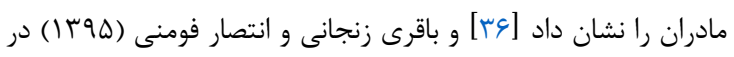

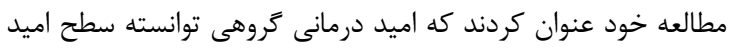

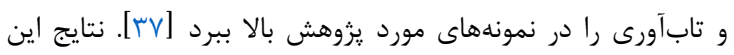

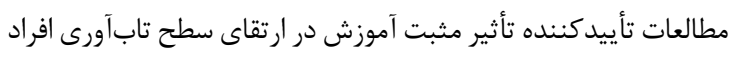

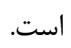

لازم به ذكر است كه دو روز در هفته به يرسش و ياسخ در رابطه با

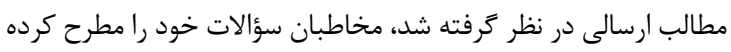

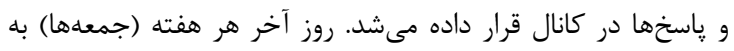

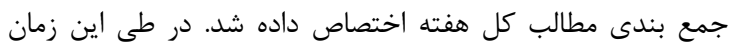

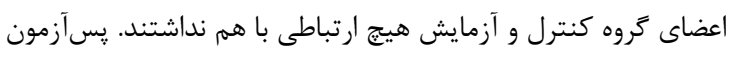

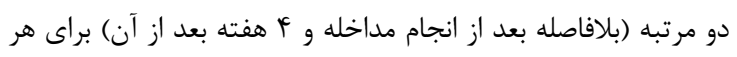

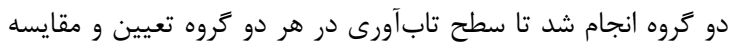

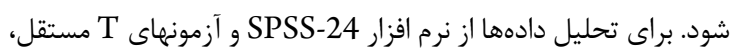
Tو زوجى و آناليز واريانس با اندازهماى تكرارى استرار استفاده شد.

\section{يافتهها}

اطلاعات مربوط به مشخصات جمعيت شناختى نمونهها نشان مى دهد

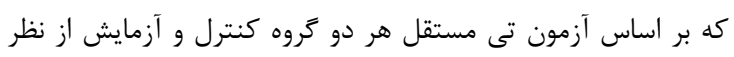

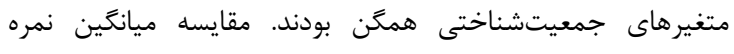

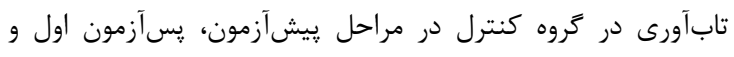

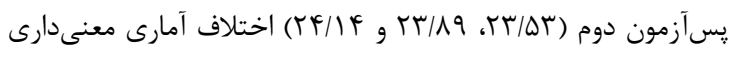

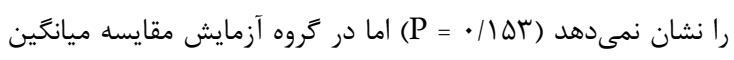

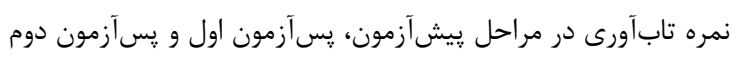

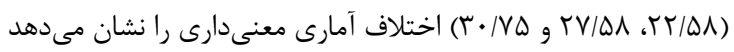

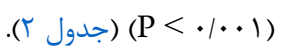

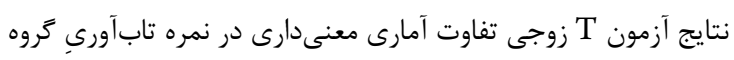

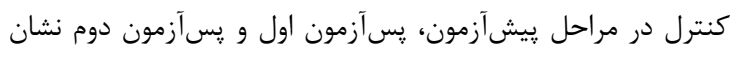

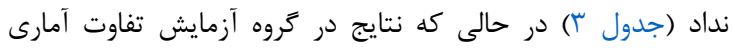
معنى دارى را نشان داد (جدول عالى).

جدول ז: ميانكَين نمره تابآورى در مراقبين خانوادَى مددجويان با اختلالات روانى شديد قبل و بعد از آموزش روانشناختى در دو كروه كنترل و آزمون

\begin{tabular}{|c|c|c|c|c|c|c|c|c|c|c|c|c|}
\hline \multirow{3}{*}{$\begin{array}{c}\text { P- } \\
\text { value }\end{array}$} & \multirow{3}{*}{$\begin{array}{c}\text { T- } \\
\text { Test }\end{array}$} & \multicolumn{2}{|c|}{ هس آزمون دوم } & \multirow{3}{*}{$\begin{array}{c}\text { P- } \\
\text { value }\end{array}$} & \multirow{3}{*}{$\begin{array}{c}\text { T- } \\
\text { Test }\end{array}$} & \multicolumn{2}{|c|}{ يس آزمون اول } & \multirow{3}{*}{$\begin{array}{c}\text { P- } \\
\text { value }\end{array}$} & \multirow{3}{*}{$\begin{array}{c}\text { T- } \\
\text { Test }\end{array}$} & \multicolumn{2}{|c|}{ بيش آزمون } & \\
\hline & & تروه & كروه & & & كروه & تروه & & & تروه & تروه & \\
\hline & & آزمون & كنترل & & & آزمون & كنترل & & & آزمون & كنترل & \\
\hline$<\cdot / \cdot \cdot \mid$ & $\varphi / Q F$ & & & $<\cdot / \cdot \cdot r$ & $r / T V$ & & & $\cdot / 49$ & - NQ & & & تاب آورى \\
\hline & & $\pm r \cdot / v \Delta$ & $\pm r \varphi / l \varphi$ & & & $\pm r V / \Delta \Lambda$ & $\pm r r / \wedge q$ & & & $\pm r r / \Delta \Lambda$ & $\pm r r / \Delta r$ & ميانكين \\
\hline & & $r / 9 r$ & $F / \wedge \varphi$ & & & $F / v q$ & F/VA & & & $\Delta / \Lambda r$ & $\Delta / 19$ & انحراف معيار \\
\hline
\end{tabular}


جدول rا: دامنه ميانكَين و انحراف معيار تابآورى و نتايج آزمون مقايسه ميانكَين در مراحل بيش آزمون، يّ آزمون اول و يس آزمون دوم در كروه كنترل

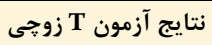

\begin{tabular}{|c|c|c|c|c|c|c|}
\hline & نتايج آزمون T زوجى & & & & & \\
\hline هِ آزمون اول و پِ & بِيش آزمون و يس آزمون & پِيش آزمون و يس آزمون & " دوم آزمون & يس آزمون & يِيش آزمون & \\
\hline \multirow[t]{3}{*}{$P=\cdot|r|, \mathrm{t}=\cdot \mid \Lambda r$} & $P=\cdot / \cdot \Delta, \mathrm{t}=r / \cdot r$ & $\mathrm{P}=\cdot / r \mathrm{r}=1 / \cdot V$ & & & & تاب آورى \\
\hline & & & $\pm r F / l F$ & $\pm r r / \wedge q$ & $\pm r r / \Delta r$ & ميانكَين \pm انحراف \\
\hline & & & $\varphi / \Lambda \varphi$ & F/v & $0 / 19$ & معيار \\
\hline
\end{tabular}

جدول f: دامنه ميانكَين و انحراف معيار تابآورى و نتايج آزمون مقايسه ميانكين در مراحل بيش آزمون، بِ آزمون اول و بِ آزمون دوم در كروه آزمون

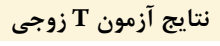

يس آزمون اول و هِ آزمون دوم آزمون الو

$\mathrm{P}<\cdot / \cdot$. I $\mathrm{t}=\mathrm{N} / \mathrm{\Lambda}$.
بيش آزمون و ها يس آزمون

دوم

$\mathrm{P}<\cdot / \cdot \cdot 1 \mathrm{t}=|r / T|$
ييش آزمون و يس آزمون اول $P<\cdot / . .1 ، \mathrm{t}=9 / 1 \Delta$

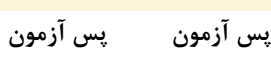

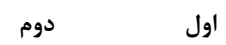

I

\begin{tabular}{|c|c|c|}
\hline $\pm r \cdot / v \Delta$ & $\pm r V / \Delta \Lambda$ & $\pm r r / \Delta \Lambda$ \\
\hline & fovg & $\Delta / \Lambda r$ \\
\hline
\end{tabular}

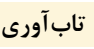
معيار

جدول ه: ميانكَين تغييرات نمره تابآورى در دو كروه آزمون و كنترل و نتايج آزمون مقايسه

\begin{tabular}{|c|c|c|c|c|c|c|}
\hline \multirow[t]{2}{*}{ P-value } & \multirow[t]{2}{*}{ T-Test } & \multicolumn{2}{|c|}{ كروه آزمون } & \multicolumn{2}{|c|}{ كروه كنترل } & \\
\hline & & انحراف معيار & ميانغين & انحراف معيار & ميانغين & \\
\hline$<\cdot|\cdot \cdot|$ & 111.4 & $r / V$ & $N / r$ & $1 / 11$ & .191 & مقياس تاب آورى \\
\hline
\end{tabular}

\section{نتيجه تيرى}

يافتههاى مطالعه حاضر قبل از اجراى مداخله نشان داد كه سطح

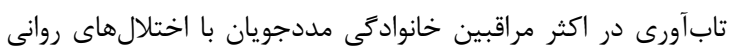

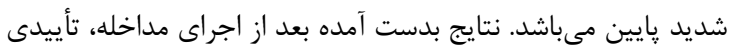

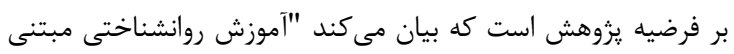

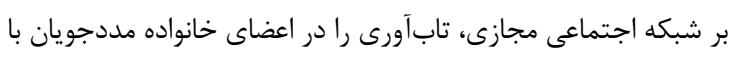

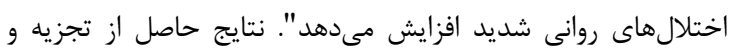

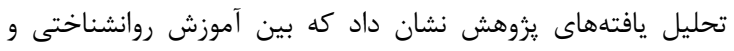

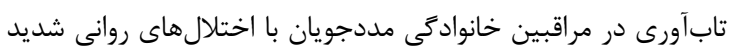

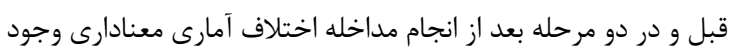

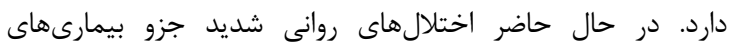

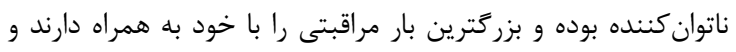

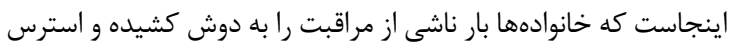

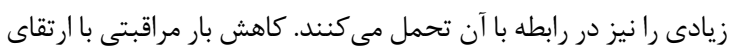
سطح تابآورى مراقبين هم در سلامت جسمى و روانى آنها تأثيركذار

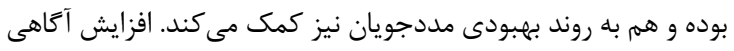

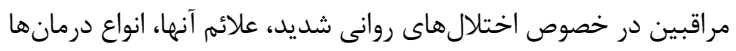

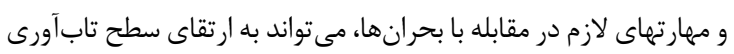

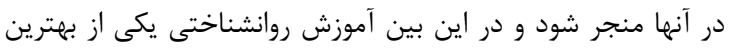

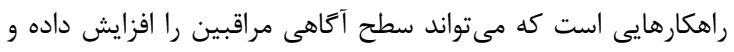

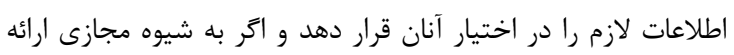

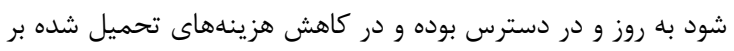
خانوادهها نيز تأثير كذار است.

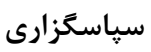

اين مقاله بخشى از نتايج باياننامه كارشناسى ارشد رشته روانير برستارى

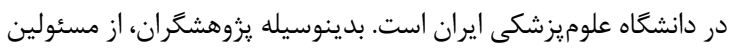

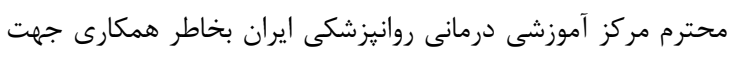

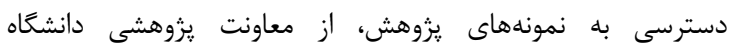

مداخلات خانوادگى بخش مهمى از روشهاى درمان مدرن مىباشد كه

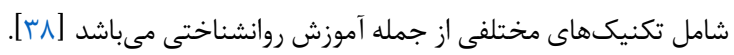

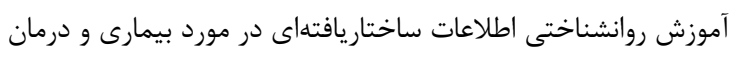

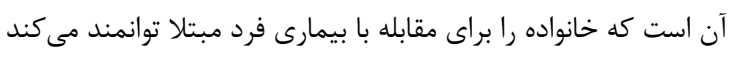

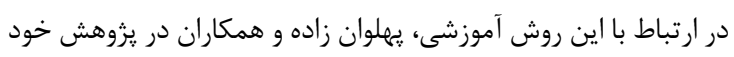

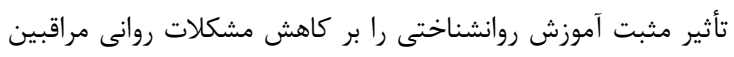

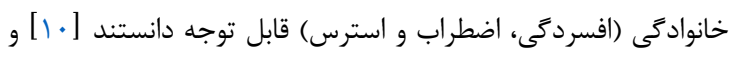

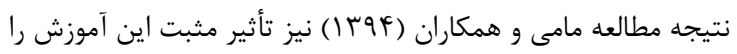

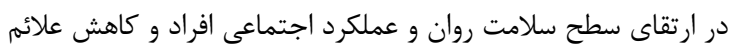

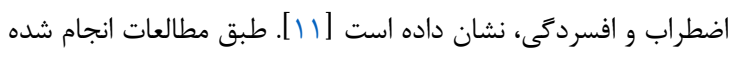

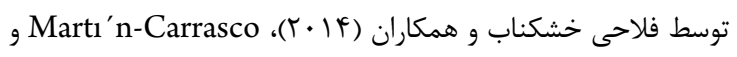

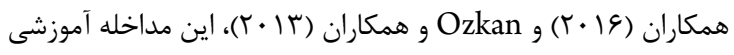

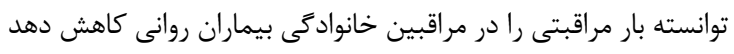

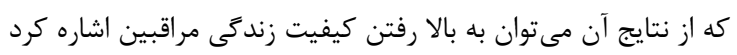

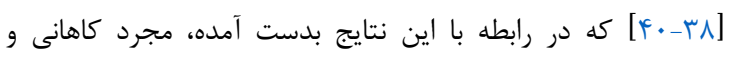

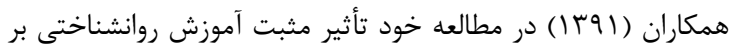

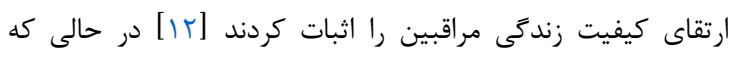

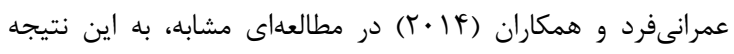

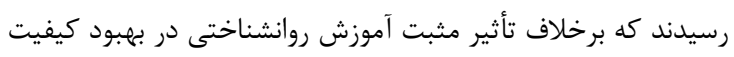

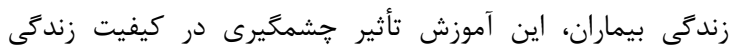

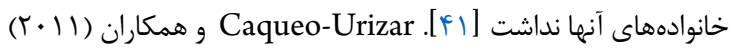

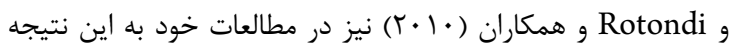

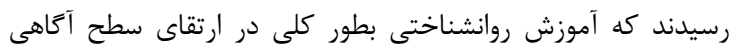

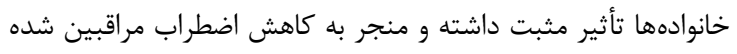

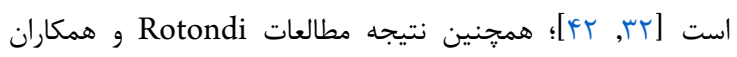

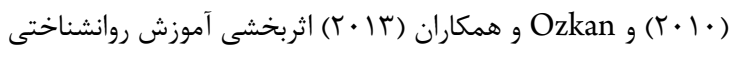

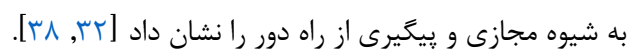




$$
\begin{aligned}
& \text { تضاد منافع } \\
& \text { نويسندگان مقاله اعلام مى دارند كه در نغارش اين مقاله هيجَّونه تضاد } \\
& \text { منافع وجود ندارد. }
\end{aligned}
$$

\section{References}

1. APA. Diagnostic and Statistical Manual of Mental Disorders DSM-5. 5th ed. United States: American Psychiatric Publishing; 2013 2013. 947 p.

2. Hajebi A, Sharifi V, Ghadiri Vasfi M, Moradi-Lakeh M, Tehranidoost M, Yunesian M, et al. A multicenter randomized controlled trial of aftercare services for severe mental illness: study protocol. BMC Psychiatry. 2013;13(1):178. doi: 10.1186/1471-244X-13-178 pmid: 23816199

3. Juan-Porcar M, Guillamon-Gimeno L, Pedraz-Marcos A, Palmar-Santos AM. Family care of people with severe mental disorders: an integrative review. Rev Lat Am Enfermagem. 2015;23(2):352-60. doi: 10.1590/01041169.0138.2562 pmid: 26039308

4. WHO. The World Health Report 2001: Mental Health : New Understanding, New Hope. France: World Health Organization; 2001

5. Farhoudian A, Sharifi V, Amini H, Basirnia A, Mesgarpour $B$, Mansouri N, et al. Prevalence of psychiatric disorders in Iran: A systematic review. Iran J Psychiatry. 2007;2:13750.

6. Mohammadi MR, Davidian H, Noorbala AA, Malekafzali $H$, Naghavi HR, Pouretemad HR, et al. An epidemiological survey of psychiatric disorders in Iran. Clin Pract Epidemiol Ment Health. 2005;1(1):16. doi: 10.1186/1745-0179-1-16 pmid: 16185355

7. Jain MA, Singh DC. Resilience and quality of life in caregivers of schizophrenia and bipolar disorder patients. Global J Hum Soc Sci Res. 2014;14(5):24-8.

8. Zauszniewski JA, Bekhet AK. Factors associated with the emotional distress of women family members of adults with serious mental illness. Arch Psychiatr Nurs. 2014;28(2):102-7. doi: 10.1016/j.apnu.2013.11.003 pmid: 24673783

9. Saunders JC. Families living with severe mental illness: a literature review. Issues Ment Health Nurs. 2003;24(2):175-98. pmid: 12554427

10. Pahlavanzadeh S, Navidian A, Yazdani M. The effect of psycho-education on depression, anxiety and stress in family caregivers of patients with mental disorders. J Kermanshah Univ Med Sci (Behbood). 2010;14(3 (47)):228 To 36 . .

11. Mami s, Kaikhavani s, Amirian k, Neyazi e. The effectiveness of family psychoeducation (atkinson and coia model) on mental health family members of patients with psychosis. J Ilam Univ Med Sci. 2016;24(1):8-17. doi: 10.18869/acadpub.sjimu.24.1.8

12. Mojarrad KAH, Ghanbari HABA, Modares GM. The efficacy of group psycho educational interventions in promoting quality of life and quality of relationships on family of patients with bipolar disorders. J Res Behav Sci. 2012;10(2):114-23.

13. Navidian A, Kermansaravi F, Rigi SN. The effectiveness of a group psycho-educational program on family caregiver burden of patients with mental disorders. BMC Res Notes. 2012;5(1):399. doi: 10.1186/1756-0500-5-399 pmid: 22853873

$$
\begin{aligned}
& \text { علوميزشكى ايران به خاطر حمايت مالى اين يروزه و از خانوادههاى }
\end{aligned}
$$

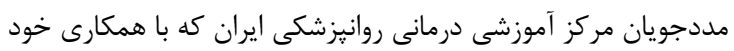

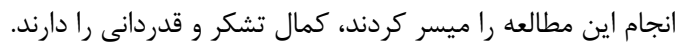

14. Mohammed S, Priya SS, George C. Caregiver burden in a community mental health program-a cross sectional study. Kerala J Psychiatry. 2015;28(1):26-33.

15. Gavois H, Paulsson G, Fridlund B. Mental health professional support in families with a member suffering from severe mental illness: a grounded theory model. Scand J Caring Sci. 2006;20(1):102-9. doi: 10.1111/j.1471-6712.2006.00380.x pmid: 16489966

16. Kaakinen JR, Coehlo DP, Steele R, Tabacco A, Hanson $\mathrm{SMH}$. Family health care nursing: Theory, practice, and research: FA Davis; 2014.

17. Shamsaei F, Cheraghi F, Esmaeilli R. The Family Challenge of Caring for the Chronically Mentally Ill: A Phenomenological Study. Iran J Psychiatry Behav Sci. 2015;9(3):e1898. doi: 10.17795/ijpbs-1898 pmid: 26576169

18. Sharifi M, Fatehizade M. Correlation between religious coping with deppression and caregiver burnout in family caregivers. Mod Car J. 2012;9(4):327-35.

19. Bambara JK, Owsley C, Wadley V, Martin R, Porter C, Dreer LE. Family caregiver social problem-solving abilities and adjustment to caring for a relative with vision loss. Invest Ophthalmol Vis Sci. 2009;50(4):1585-92. doi: 10.1167/iovs.08-2744 pmid: 19060279

20. Kirmani MN, Sharma P, Anas M, Sanam R. Hope, resilience and subjective well-being among college going adolescent girls. Int J Hum Soc Sci Stud 2015;2(1):26270.

21. Duggal D, Sacks-Zimmerman A, Liberta T. The Impact of Hope and Resilience on Multiple Factors in Neurosurgical Patients. Cureus. 2016;8(10):e849. doi: 10.7759/cureus.849 pmid: 27909637

22. Zauszniewski JA, Bekhet AK, Suresky MJ. Resilience in family members of persons with serious mental illness. Nurs Clin North Am. 2010;45(4):613-26, vii. doi: 10.1016/j.cnur.2010.06.007 pmid: 20971340

23. Macleod SH, Elliott L, Brown R. What support can community mental health nurses deliver to carers of people diagnosed with schizophrenia? Findings from a review of the literature. Int J Nurs Stud. 2011;48(1):10020. doi: 10.1016/j.ijnurstu.2010.09.005 pmid: 20956000

24. Glynn SM, Cohen AN, Niv N. New challenges in family interventions for schizophrenia. Expert Rev Neurother. 2007;7(1):33-43. doi: 10.1586/14737175.7.1.33 pmid: 17187495

25. Shimazu K, Shimodera S, Mino Y, Nishida A, Kamimura N, Sawada K, et al. Family psychoeducation for major depression: randomised controlled trial. Br J Psychiatry. 2011;198(5):385-90. doi: 10.1192/bjp.bp.110.078626 pmid: 21343330

26. Bhattacharjee D, Rai AK, Singh NK, Kumar P, Munda SK, Das B. Psychoeducation: A Measure to Strengthen Psychiatric Treatment. Delhi Psychiatry J. 2011;14(1):33-9.

27. Sauceda M. Family Psychoeducation for Latino Populations with Mental Illness Living in the United States. [Thesis]. In press 2011. 
28. Sin J, Jordan CD, Barley EA, Henderson C, Norman I. Psychoeducation for siblings of people with severe mental illness. Cochrane Database Syst Rev. 2015(5):CD010540. doi: 10.1002/14651858.CD010540.pub2 pmid: 25953641

29. Bazi A, Khosravi Bonjar A, Saravani-Aval S, SalehMoghadam AR, Delaramnasab M, Shahdadi H, et al. The Effect of E-Learning on Adherence to the Therapeutic Regimen in Patients with Type II Diabetes. J Diabetes Nurs. 2017;5:95-109.

30. Salehmoghaddam AR, Khosravi Bonjar A, Karimi Moonaghi H, Gholami H. An Investigation of The Effect of E-learning Education Method on Dietary Regimen in Type 2 Diabetic Patients Evid Based Car J. 2013;3(3):518.

31. Hosseinzadeh Z, Kadivar M, Seyedfatemi N, Zolfaghari M, Mehran A. The Impact of Virtual-Based Education on Nurses' Psychological Empowerment in the Level II Neonatal Care Unit. Iranian J Med Educ. 2017; 17:102-15.

32. Rotondi AJ, Anderson CM, Haas GL, Eack SM, Spring $\mathrm{MB}$, Ganguli R, et al. Web-based psychoeducational intervention for persons with schizophrenia and their supporters: one-year outcomes. Psychiatr Serv. 2010;61(11):1099-105.

doi: 10.1176/ps.2010.61.11.1099 pmid: 21041348

33. Connor KM, Davidson JR. Development of a new resilience scale: the Connor-Davidson Resilience Scale (CD-RISC). Depress Anxiety. 2003;18(2):76-82. doi: 10.1002/da.10113 pmid: 12964174

34. Mohammadi M. The study of key factors influences on resiliency of substance abuse at risk. Tehran Tehran University; 2005.

35. Keyhani M, Taghvaei D, Rajabi A, Amirpour B. Internal Consistency and Confirmatory Factor Analysis of the Connor-Davidson Resilience Scale (CD-RISC) among Nursing Female Students. Iran J Med Educ. 2015;14(10):857-65.
36. Nikrah N, Zare M, Khakbaz H. Effectiveness of Social Problem-Solving Group Training on Increasing the Resiliency of Mothers with Addicted Choldren. Soc Welfare. 2015;15:185-99.

37. Bagheri Zanjani Asl Monfared L, Entesar Foumany G. The effectiveness of group based hope-therapy on increasing resilience and hope in life expectancy in patients with breast cancer. J Health Promot Manage. 2016;5(4):56-62.

38. Ozkan B, Erdem E, Demirel Ozsoy S, Zararsiz G. Effect of psychoeducation and telepsychiatric follow up given to the caregiver of the schizophrenic patient on family burden, depression and expression of emotion. PakJ Med Sci. 2013;29(5):1122-7. pmid: 24353704

39. Fallahi Khoshknab M, Sheikhona M, Rahgouy A, Rahgozar M, Sodagari F. The effects of group psychoeducational programme on family burden in caregivers of Iranian patients with schizophrenia. J Psychiatr Ment Health Nurs. 2014;21(5):438-46. doi: 10.1111/jpm. 12107 pmid: 23980535

40. Martin-Carrasco M, Fernandez-Catalina P, DominguezPanchon AI, Goncalves-Pereira M, Gonzalez-Fraile E, Munoz-Hermoso P, et al. A randomized trial to assess the efficacy of a psychoeducational intervention on caregiver burden in schizophrenia. Eur Psychiatry. 2016;33:9-17. doi: 10.1016/j.eurpsy.2016.01.003 pmid: 26852375

41. Omranifard V, Yari A, Kheirabadi GR, Rafizadeh M, Maracy MR, Sadri S. Effect of needs-assessment-based psychoeducation for families of patients with schizophrenia on quality of life of patients and their families: A controlled study. J Educ Health Promot. 2014;3:125. doi: 10.4103/2277-9531.145937 pmid: 25540798

42. Caqueo-Urízar A, Gutiérrez-Maldonado J, PalmaFaúndez C. Caregivers of patients with schizophrenia: how much they know and learn after a psychoeducational intervention? Proc Soc Behav Sci. 2011;30:2468-76. doi: 10.1016/j.sbspro.2011.10.482 